\title{
Effect of morphology and defect density on electron transfer of electrochemically reduced graphene oxide
}

\author{
Yan Zhang å, Huilian Hao ${ }^{\mathrm{a}}$, and Linlin Wang ${ }^{\mathrm{b}}$
}

${ }^{a}$ School of Material Engineering, Shanghai University of Engineering Science, Shanghai 201620,

P.R. China

${ }^{\mathrm{b}}$ College of Chemistry and Chemical Engineering, Shanghai University of Engineering Science,

Shanghai 201620, P.R. China

${ }^{\S}$ Corresponding author: yanzhang@sues.edu.cn

Email addresses:

YZ: yanzhang@sues.edu.cn

HH: huilian.hao@sues.edu.cn

LW: wlinlin@mail.ustc.edu.cn 


\section{ABSTRACT}

Electrochemically reduced graphene oxide (ERGO) is widely used to construct electrochemical sensors. Understanding the electron transfer behavior of ERGO is essential for its electrode material applications. In this paper, different morphologies of ERGO were prepared via two different methods. Compared to ERGO/GCEs prepared by electrochemical reduction of pre-deposited GO, more exposed edge planes of ERGO are observed on the surface of ERGO-GCE that was constructed by electrophoretic deposition of GO. The defect densities of ERGO were controlled by tuning the mass or concentration of GO. The electron transfer kinetics $\left(k^{0}\right)$ of GCE with different ERGOs was comparatively investigated. Owing to increased surface areas and decreased defect density, the $k^{0}$ values of ERGO/GCE initially increase and then decrease with incrementing of GO mass. When the morphology and surface real areas of ERGO-GCE are the same, an increased defect density induces an accelerated electron transfer rate. $k^{0}$ valuesof ERGO-GCEs are about 1 order of magnitude higher than those of ERGO/GCEs due to the difference in the amount of edge planes. This work demonstrates that both defect densities and edge planes of ERGO play crucial roles in electron transfer kinetics.

Keywords: electrochemically reduced graphene oxide; graphene; electron transfer; defect density; edge plane 


\section{Introduction}

Graphene, a two-dimensional single layer crystal of $s p^{2}$-hydridized carbon atoms has attracted much attentions as an electrode material due to its spectacular physical, chemical, and electrical properties [1]. More and more sensors, biosensing devices, supercapacitors and batteries based on graphene[2], metallic and bimetallic nanoparticles decorated graphene oxide or graphene have been developed[3-19]. Up to now, chemically reduced graphene oxide (CRGO) has been widely used to fabricate graphene electrodes [20]. Different chemical regents such as L-ascorbic acid [21], hydrazine hydrate [22], and hydroxylamine [23] have been used to synthesize CRGO. Compared to synthesis of CRGO, the electrochemical reduction method provides a facile, fast, scalable, economic and environmentally friendly pathway to the production of graphene materials [24]. Two different electrochemical reduction approaches were used to synthesize electrochemically reduced graphene oxide (ERGO): a one-step approach that involves direct electrochemical deposition of graphene in GO suspension onto an electrode surface, and a two-step approach that requires pre-deposition of GO onto the surface of an electrode to induce electrochemical reduction $[25,26]$. The influencing factors for synthesis of ERGO, such as the optimization of $\mathrm{pH}$ value [27], different cycle numbers of performing cyclic voltammetry (CV) [27], and applied potential [28, 29] also have been studied. ERGO has been extensively explored as a candidate material for electrodes and their modification [30]. Hamsawahini et al. [31] reported that ERGO-modified electrodes exhibited a good sensitivity of $\mathrm{Pb}$ (II) ions. Sun et al. [32] fabricated an electrochemical DNA biosensor for the detection of the ssDNA sequence with an ERGO electrode. ERGO electrodes also exhibited significantly enhanced catalytic activity toward the oxygen reduction reaction (ORR) in an alkaline medium compared with the initial GO electrode[29]. ERGO-based electrodes have drawn considerable attention due to the simplicity of their preparation process and outstanding electrochemical properties.

Understanding the electron transfer (ET) rate kinetics of graphene is essential for exploring its application as an electrode material. The studies of graphene's ET rate were mostly focused on CRGO-modified electrodes [33] and monolayer graphene [34]. Up to now, the corresponding theory has not been developed. Hallam et al. found that the edge plane of graphene is the origin of electron transfer rather than the basal plane[35]. Zhong et al. demonstrated that the electrochemical activity of single layer graphene depends strongly on its defect density [36]. 
However, Güell et al. reported that no increase of the electron transfer rate was observed at the edges or steps in comparison to the basal plane[37]. Velicky et al. found that the basal planes of mono- and multilayer graphene exhibit significant electron transfer activity and changes in kinetics[34]. Only a few of studies have focused on the electron transfer of ERGO. Liu et al. reported that the glass carbon electrode (GCE) modified by reductively deposited graphene with its standing configuration is beneficial to electron transfer compared with its tiled configuration [38]. But this fact is not sufficient to understand the electron transfer of ERGO. Herein, we investigated the impact of morphology and defect density on electron transfer of ERGO. The different morphologies and defect densities of ERGOs were prepared using different amount and concentration of GO via two different approaches, and characterized by scanning electron microscopy, x-ray photoelectron spectroscopy, and Raman spectroscopy. The electron transfer kinetics $\left(k^{0}\right)$ of ERGO electrodes were investigated using $\mathrm{Fe}(\mathrm{CN})_{6}^{3-14-}$, and the results suggest that $k^{0}$ value is closely related to both the morphology and defect density of graphene; and a high defect density and more exposed edge planes induce acceleration of electron transfer.

\section{Experimental}

\subsection{Materials}

All chemicals were analytical grade and were purchased from Sinopharm (Shanghai, China). All aqueous solutions were prepared using ultrapure water $(18.2 \mathrm{M} \Omega \mathrm{cm})$. A phosphate buffer solution (PBS) containing $\mathrm{K}_{2} \mathrm{HPO}_{4}$ and $\mathrm{KH}_{2} \mathrm{PO}_{4}$ was adjusted to the desired $\mathrm{pH}$. GO was prepared using natural graphite powder using a modified Hummer's method that was described in detail in previous works [21].

\subsection{Electrochemically Reduced Graphene Oxide}

Prior to electrochemical reduction, the GC electrode ( $3 \mathrm{~mm}$ in diameter) was polished with 0.3 and $0.05 \mu \mathrm{m}$ alumina powders and then sonicated in distilled water and anhydrous ethanol as described in our previous works [20]. The electrochemical reduction of graphene oxide was carried out using two methods.

The first method was electrochemical reduction of GO-modified GCE which required pre-deposition of GO onto a GCE substrate prior to processing. GO/GCE was achieved through a 
solution casting procedure. Typically, a $6 \mu \mathrm{L}$ portion of GO aqueous suspension with different concentrations $(0.1,0.5,1.0$, and $2.0 \mathrm{mg} / \mathrm{mL})$ was dropped at the center of pretreated GCE, and dried under high vacuum. The electrochemical reductions were performed on a $\mathrm{CHI} 660 \mathrm{C}$ electrochemical workstation (Chen Hua Co., China) using a three-electrode system, with GO/GC as the working electrode, a platinum wire as the counter electrode, and a saturated calomel electrode as the reference electrode. The electrolyte solution was purged with pure $\mathrm{N}_{2}$ to remove oxygen for $10 \mathrm{~min}$ before the electrochemical reduction. Cyclic voltammetry was performed on the GO-modified GCE in $0.1 \mathrm{M}, \mathrm{pH}$ 9.23 PBS. The potential window was applied from -1.5 to 0.5 $\mathrm{V}$, and the scan rate was $50 \mathrm{mV} / \mathrm{s}$. After 10 reduction cycles were applied, the electrochemically reduced graphene oxide modified electrode was obtained and designated ERGO/GCE. The ERGO/GCE was rinsed with deionized (DI) water three times and then dried at room temperature.

The other electrochemical reduction method used was electrophoretic deposition of graphene. The experimental conditions were the same as in the first method, except that a bared GCE was used as the working electrode and the electrolyte solution was the GO solution (in $0.1 \mathrm{M}, \mathrm{pH} 9.23$ PBS). After 10 reduction cycles were applied, the electrochemically reduced graphene oxide modified GCE was obtained and designated ERGO-GCE. The ERGO-GCE was rinsed with DI water three times and then dried at room temperature.

\subsection{Characterization}

Scanning electron microscope (SEM) images of the modified GC electrode were acquired on an Ultra 55 field-emission scanning electron microscope (Zeiss, Germany) with $3 \mathrm{kV}$ of acceleration voltage.

The Raman spectra were recorded using an InVia-Reflex Raman spectrometer (Renishaw, England) with a 532-nm laser source.

The GOs and as-prepared ERGO were characterized by using x-ray photoelectron spectroscopy (XPS). XPS were collected from a glassy carbon substrate on an AXIS Ultra spectrometer (Kratos Analytical Inc., United Kingdom) with a monochromated Al $K \alpha$ source. A survey scan was performed from 0 to $1200 \mathrm{eV}$ on the samples, followed by a narrow scan on the $\mathrm{C}$ $1 s$ region of the spectrum. Peak fit analyses were performed specifically on the $\mathrm{C} 1 s$ region of the narrow scan using CasaXPS software. 


\section{Results and Discussion}

ERGO films were prepared by cyclic voltammetry in $0.1 \mathrm{M} \mathrm{pH} 9.23$ phosphate buffer with a potential window from -1.5 to $0.5 \mathrm{~V}$. Figure 1 depicts the $\mathrm{CV}$ profile of electrodes modified with different GO masses. An obvious cathodic peak at $-1.2 \mathrm{~V}$, and a pair of redox peaks at approximately -0.3 and $-0.2 \mathrm{~V}$ are observed [Fig. 1(a)]. From the inset of Fig. 1(a), the currents of redox peaks at -0.3 and $0.2 \mathrm{~V}$ initially decrease and then remain stable after several potential scans. This is in agreement with previously reported results [39], suggesting that the stable oxygen-containing groups cannot be easily and completely reduced [40,41]. With an increase of GO mass from 0.6 to $3 \mu \mathrm{g}$, the cathodic peak current increases and the position of the reduction peak at $-1.2 \mathrm{~V}$ shifts toward higher negative potential, resulting from the growth of functional oxygen groups. Once the amount of GO was increased to 6 or $12 \mu \mathrm{g}$, the redox peaks cannot be clearly seen. A very broad reduction peak is presented at $-1.5 \mathrm{~V}$ and the peak current continuously diminished with successive potential scans [Figs. 1(c) and 1(d)]. It is suggested that the electrochemical reduction rate decreased with the increase of GO mass. As is known, with the incrementing of GO mass, the increased functional oxygen groups may block the electron pathways [30], thus resulting in a decreasing reduction rate.

In order to quantify the degree of reduction, as-prepared samples were investigated by XPS. Figure 2 shows the high-resolution $\mathrm{C} 1 s$ spectra of the GO and ERGOs, including curve fits for the various carbon components. In addition, the percentages of functional groups and the $\mathrm{sp}^{2}$ fraction were calculated from XPS spectra for a better comparison. In Fig. 2(a), the peaks of hydroxyl, carbonyl, and carboxyl groups in GOs appear at 286.9, 288.1 and $291.5 \mathrm{eV}$, respectively [42]. Upon electrochemical reduction, the graphitic $s p^{2}$-carbon concentration increased and the intensities of these peaks in ERGOs were significantly reduced. From these results, it can be confirmed that the GO has been effectively reduced during the CV cycles. As seen in Fig. 2(b), the graphitic $s p^{2}$-carbon concentration increased to approximately $58.45 \%$ for the resulting ERGO $(0.6 \mu \mathrm{g})$, and the percentage of $\mathrm{C}=\mathrm{O}$ and $\mathrm{O}-\mathrm{C}=\mathrm{O}$ groups declined obviously, while there are still considerable numbers of $\mathrm{C}-\mathrm{O}$ groups remaining unremoved, which is consistent with literature reports $[39,41]$. These results indicate that carbonyl and carboxyl groups can be easily converted at $-1.2 \mathrm{~V}$ [39], and that the cathodic peak disappears in the second 
cycle, further indicating that carbonyl and carboxyl groups can be easily and quickly reduced. As the mass of the GOs increased from 0.6 to $2.0 \mu \mathrm{g}$, the percentage of $\mathrm{C}=\mathrm{O}$ and $\mathrm{C}-\mathrm{O}$ groups increased, thus indicating that more residual oxygen functional groups remained.

The surface morphology of ERGO/GCE was elucidated by SEM. As shown in Fig. 3(a), a thin, restacking ERGO coating layer can be clearly observed on the GCE surface. Owing to the excellent dispersion of GO sheets and $\pi-\pi$ interaction, ERGO sheets prepared via electrochemical reduction of the GO layer were prone to overlap with each other and they thus successfully covered the surface of GCE.

Raman spectroscopy is a very useful technique for characterizing the structure of carbon material. Fig. 3(b) shows the Raman spectra of GO and ERGO samples. Using 532-nm laser excitation, the $D$ and $G$ bands are represented by peaks at approximately 1345 and $1590 \mathrm{~cm}^{-1}$, which correspond to $s p^{2}$ and $s p^{3}$ carbon stretching modes, respectively [22]. Owing to the introduction of defects by the fast reduction rate [40], the intensity of the $D$ band of ERGO increased compared with that of GO, implying that the GOs have been successfully reduced. The ratio of the $D$ and $G$ band intensity $\left(I_{D} / I_{G}\right)$ is a measure of the amount of disorder present within the material. As shown in Fig. 3(b), the $I_{D} / I_{G}$ ratios of ERGOs are higher than that of GO, indicating an increase in the number of smaller $s p^{2}$ domains after electrochemical reduction. The $I_{D} / I_{G}$ ratios of ERGOs decrease concomitantly with an increasing amount of GO, resulting from the decrease in the electrochemical reduction rate. The decrease in $I_{D} / I_{G}$ ratio suggests a lower defect density upon introduction of ERGO.

To explore the electrochemical performance of ERGO/GCE, $\mathrm{Fe}(\mathrm{CN}) 6^{3-14-}$ which is sensitive to the surface chemistry of carbon-based electrodes[34, 43], was used as an out-sphere electron transfer redox probe. In comparison with the CV response of bare GCE, larger peak current responses and narrow peak-to-peak potential separations $\left(\Delta E_{P}\right)$ are observed with that of ERGO/GCE in Fig. 4. The larger current responses of $\mathrm{Fe}(\mathrm{CN}) 6^{3-/ 4-}$ indicate an increase in the number of electrochemically active sites [39]. As we know, electrochemically active sites result from excellent conductivity and large surface area $[44,45]$. With increasing GO mass, the surface areas of ERGO and the residual oxygen functional groups were increased. Note that the conductivity of ERGO films was impeded by the increased percentage of residual oxygen functional groups. Hence, the intensity of the peak current and electrochemically active sites of 
ERGOs initially increased and then decreased, resulting from the combined effect of increased surface area and decreased conductivity.

Table 1. Peak potential separation and ET rate of $\mathrm{Fe}(\mathrm{CN})_{6}{ }^{3-/ 4-}$ on different electrodes.

\begin{tabular}{cccccc}
\hline $\begin{array}{c}\text { Electrode (two } \\
\text { step approach) }\end{array}$ & $\Delta E_{P}(\mathrm{~V})$ & $k^{\mathrm{o}}\left(10^{-5} \mathrm{~cm} \mathrm{~s}^{-1}\right)$ & $\begin{array}{l}\text { Electrode (one } \\
\text { step approach) }\end{array}$ & $\Delta E_{P}(\mathrm{~V})$ & $k^{\mathrm{o}}\left(10^{-5} \mathrm{~cm} \mathrm{~s}^{-1}\right)$ \\
\hline Bare GCE & 0.79 & 0.271 & & & \\
$\mathrm{ERGO}_{0.6} / \mathrm{GCE}$ & 0.56 & 2.542 & $\mathrm{ERGO}_{0.1}$-GCE & 0.266 & 44.495 \\
$\mathrm{ERGO}_{3} / \mathrm{GCE}$ & 0.462 & 6.60 & $\mathrm{ERGO}_{0.5}$-GCE & 0.321 & 26.04 \\
$\mathrm{ERGO}_{6} / \mathrm{GCE}$ & 0.532 & 3.339 & $\mathrm{ERGO}_{1.0}$-GCE & 0.331 & 23.631 \\
$\mathrm{ERGO}_{12} / \mathrm{GCE}$ & 0.54 & 3.089 & & & \\
\hline
\end{tabular}

We now turn to an exploration of the electron transfer kinetics of graphene, for which $\Delta E_{P}$ was greater than $150 \mathrm{mV}$. Electron transfer rate kinetics $\left(k^{0}\right)$ can be directly calculated as

$$
k^{0}=2.18\left(\frac{\alpha n F D \vartheta}{R T}\right)^{1 / 2} e^{-\left[\left(\alpha^{2} F / R T\right) n \Delta E_{P}\right]}
$$

where $\alpha$ is the transfer coefficient, $n$ the number of electrons involved in the redox reaction, $F$ the faraday constant, $D$ the diffusion coefficient, $R$ the gas constant, $T$ the absolute temperature, and $v$ the scan rate $[34,46]$. As in the case of Nicholson analysis, it was assumed that the reduction and oxidation were symmetrical, so $\alpha \approx 0.5, n=1$ (one-electron processes). The calculated values for the ET rate kinetics are presented in Table 1. The $\Delta E_{P}$ and $k^{0}$ values of $\mathrm{Fe}(\mathrm{CN}) 6^{3-14-}$ on bare GCE were $0.79 \mathrm{~V}$ and $2.71 \times 10^{-6} \mathrm{~cm} \mathrm{~s}^{-1}$, respectively. For comparison, $\mathrm{ERGO}_{0.6} / \mathrm{GCE}$ and $\mathrm{ERGO}_{3} / \mathrm{GCE} \quad\left(\mathrm{ERGO}_{0.6} / \mathrm{GCE}\right.$ and $\mathrm{ERGO}_{3} / \mathrm{GCE}$ represent the ERGOs prepared via electrochemical reduction of 0.6 and $3 \mu \mathrm{g}$ GO, respectively) both show a higher $k^{0}$ value, 25.42 $\times 10^{-6}$ and $66.0 \times 10^{-6} \mathrm{~cm} \mathrm{~s}^{-1}$, respectively. However, when the amount of GO was further increased, the $k^{0}$ values of $\mathrm{ERGO}_{6} / \mathrm{GCE}$ and $\mathrm{ERGO}_{12} / \mathrm{GCE}$ decreased to $33.39 \times 10^{-6}$ and $30.89 \times$ $10^{-6} \mathrm{~cm} \mathrm{~s}^{-1}$. This is possible due to the comprehensive effects of surface area and defect density. Both the edge planes and basal planes of ERGO would increase as the surface area increases, whether the electron transfer in graphene originates from edge planes or basal planes, resulting in higher electron transfer kinetics. Despite the defect induced decrease of conductivity[47], it has been demonstrated that increasing the defect density can lead to a higher density of states that will lead to faster kinetics [36]. Hence, the electron transfer kinetics begins to increase and then 
decreases with the incrementing of GO mass, resulting from increased surface area and decreased defect density.

Electrophoretic deposition of graphene is depicted in Fig. 5. Contrary to the CV results of GCE in a PBS without a GO solution, a new cathodic current peak of ERGO-GCE appears at approximately $-1.33 \mathrm{~V}$, resulting from the reduction of functional oxygen groups. The cathodic peak then disappears in the second cycle, demonstrating that the reduction of functional oxygen groups in GO occurs quickly and irreversibly [40]. The degrees of reduction of as-prepared samples were also investigated by XPS. The high resolution C $1 s$ spectra of ERGO produced by electrophoretic deposition are shown in Fig. 6. The peak intensities of hydroxyl, carbonyl, and carboxyl groups in ERGO films are much smaller than those in GO film, indicating considerable deoxygenation. With an increase of GO concentration, the percentages of oxygen functional groups in ERGOs increased slightly.

The morphology of ERGO deposited on GCE was also confirmed by SEM. As shown in Figs. 7(a-c), ERGO-GCE prepared by different concentrations of GO have similar morphologies. The surface of GCE is not completely covered, and flat and vertical graphene sheets with more exposed edge planes are observed on the surface of GCE, demonstrating that the ERGO was successfully deposited on the GCE.

Figure 7(d) shows Raman spectra of electrodeposited ERGO films using different concentrations of GO. In comparison with GO, the intensity of the $D$ band and the $I_{D} / I_{G}$ ratio increase upon deposition of ERGO. Owing to the fast reduction rate, an increase in the number of smaller $s p^{2}$ domains and significant disorder exist in the ERGO. With the incrementing of GO concentration, the $I_{D} / I_{G}$ ratios of ERGOs decrease from 1.792 to 1.614 , suggesting that the defect density of ERGO decreased.

The electrochemical performances of ERGO-GCE were measured, and the $I_{p c}$ and $I_{p a}$ valute of bare GCE are 2.65 and $3.45 \mu \mathrm{A}$, respectively [Fig. 8(a)]. In comparison with GCE, the peak currents of ERGO-GCE exhibit an obvious increase, suggesting a significant increase in the number of electrochemically active sites. The peak currents of different ERGO-GCEs are almost the same, indicating that they have the same electrochemically active sites. In order to explain the results, all of the real surface areas of ERGO-GCEs were calculated based on the Randles-Sevcik equation $\left(I_{p}=2.69 \times 10^{5} A n^{3 / 2} D^{1 / 2} C_{0} v^{1 / 2}\right)$ by detecting the peak current with the 
increment of scan rate increased to approximately 18 times that of bare GCE, consistent with the SEM results. The same real surface areas and good conductivity of graphene may be the reason for the same peak currents. The electron transfer kinetics were also evaluated, and compared to bare GCE, the $\Delta E_{P}$ value of $\mathrm{ERGO}_{0.1}$-GCE decreases from 0.79 to $0.266 \mathrm{~V}$ [Fig. $8(\mathrm{a})]$ and the ET rate kinetics increases from $2.71 \times 10^{-6}$ to $4.45 \times 10^{-4} \mathrm{~cm} \mathrm{~s}^{-1}$ (Table 1), demonstrating that electrophoretically deposited graphene with exposed edge planes can successfully accelerate the electron transfer rate. Upon increasing the GO concentration to 0.5 $\mathrm{mg} / \mathrm{mL}$, the ET rate kinetics decreases to $2.604 \times 10^{-4} \mathrm{~cm} \mathrm{~s}^{-1}$. When the concentration of GO was $1.0 \mathrm{mg} / \mathrm{mL}$, the ET rate kinetics decreased to $2.36 \times 10^{-4} \mathrm{~cm} \mathrm{~s}^{-1}$. It is worth noting that all of the ERGO-GCEs have the same surface areas and morphologies. The electron transfer kinetics decreased with a decreased defect density of the ERGO. Thus, this further demonstrates that the defect density has a significant positive impact on the ET rate.

Comparisons of the electrochemical performance of ERGO-modified GCE using different preparation methods are shown in Fig. 8(b). Owing to the larger surface areas, the peak currents of $\mathrm{ERGO}_{3} / \mathrm{GCE}$ are larger than those of $\mathrm{ERGO}_{0.1}$-GCE, indicating that the $\mathrm{ERGO}_{3} / \mathrm{GCE}$ has many more electrochemically active sites than that of $\mathrm{ERGO}_{0.1}$-GCE. However, the electron transfer rate of ERGO-GCE was 1 order of magnitude higher than that of ERGO/GCE as shown in Table 1. Despite ERGO $_{1.0}$-GCE having a lower defect density and higher percentages of oxygen functional groups than $\mathrm{ERGO}_{0.6} / \mathrm{GCE}$, the $k^{0}$ value of $\mathrm{ERGO}_{1.0} \mathrm{GCE}$ is much higher than that of $\mathrm{ERGO}_{0.6} / \mathrm{GCE}$. As is known, the defect density can lead to a higher density of states that will lead to faster kinetics [19]. Therefore, the defect density no longer explains these results. Considering the different morphologies of ERGO obtained via different approaches, and owing to the Van der Waals' force and $\pi-\pi$ interaction, the restacking of graphene on the ERGO/GCE results in a lower number of exposed edge planes. On the other hand, significantly more exposed edge planes have been observed on the surface of ERGO-GCE, and it is very likely that the high $k^{0}$ value of ERGO can be attributed to exposed edge planes. Thus, the increase in the number of exposed edge planes of ERGO can significantly enhance the electron transfer rate.

\section{Conclusions}


In summary, SEM, XPS, and Raman spectroscopy analyses revealed that ERGO was successfully prepared via electrochemical methods. As for the electrochemical reduction of pre-deposited GO on GCE, with increasing GO mass the electrochemical reduction rate decreased, which may induce a decrease of the defect density. The electron transfer rate kinetics of ERGO/GCE begin to increase and then decrease with incrementing of GO mass. This is attributed to the comprehensive effects of the increased surface area and decreased defect density. As for electrophoretic deposition of GO, ERGO-GCEs prepared in different GO concentrations have the same real surface areas, morphology, and more exposed edge planes. With the increment of GO concentrations, owing to the decrease of defect density, the electron transfer rate of ERGO-GCE was decelerated. Compared to different ERGO electrodes, the electron transfer rate of ERGO-GCE with more exposed edge planes is significantly higher than that of ERGO/GCE. It is clear that the number of exposed edge planes and defect density have positive effects on the electron transfer of graphene. These considerations are crucial for the development of graphene electrode applications.

\section{Acknowledgement}

This work was supported by the Shanghai "Sailing Program" foundation (Grants Nos. 14YF1409500 and 14YF1409700), the Foundation of Shanghai Young Teachers in Universities or Colleges (Grant No. ZZgcd14011), and the Natural Science Foundation of China (Grants Nos. 51602192 and 11504229).

\section{References}

[1] A.K. Geim, K.S. Novoselov, The Rise of Graphene, Nat. Mater. 6 (2007) 183-191.

[2] Y. Shao, J. Wang, H. Wu, etc., Graphene Based Electrochemical Sensors and Biosensors: A Review, Electroanal. 22 (2010) 1027-1036.

[3] O. Akyıldırım, H. Medetalibeyoğlu, S. Manap, M. Beytur, F.S. Tokalı, M.L. Yola, N. Atar, Electrochemical Sensor Based on Graphene Oxide/Iron Nanoparticles for the Analysis of Quercetin, Int. J. Electrochem. Sci. 10 (2015) 7743-7753.

[4] V.K. Gupta, M.L. Yola, N. Atar, Z. Üstündağ, A.O. Solak, Electrochemical studies on graphene oxide-supported metallic and bimetallic nanoparticles for fuel cell applications, J. Mol. Liq. 191 (2014) 172-176. 
[5] N. Atar, T. Eren, M.L. Yola, H. Gerengi, S. Wang, Fe@Ag nanoparticles decorated reduced graphene oxide as ultrahigh capacity anode material for lithium-ion battery, Lonics 21 (2015) 3185-3192.

[6] N. Atar, T. Eren, M.L. Yola, H. Karimi-Maleh, B. Demirdogen, Magnetic iron oxide and iron oxide@gold nanoparticle anchored nitrogen and sulfur-functionalized reduced graphene oxide electrocatalyst for methanol oxidation, RSC Adv. 5 (2015) 26402-26409.

[7] M.L. Yola, V.K. Gupta, N. Atar, New molecular imprinted voltammetric sensor for determination of ochratoxin A, Adv. Mater. Sci. Eng. C, 61 (2016) 368-375.

[8] M.L. Yola, T. Eren, N. Atar, A novel and sensitive electrochemical DNA biosensor based on Fe@Au nanoparticles decorated graphene oxide, Electrochim. Acta 125 (2014) 38-47.

[9] G. Kotan, F. Kardas, O.A. Yokus, O. Akyldrm, H. Saral, T. Eren, M.L. Yola, N. Atar, A novel determination of curcumin via Ru@Au nanoparticle decorated nitrogen and sulfur-functionalized reduced graphene oxide nanomaterials, Anal. Methods 8 (2016) 401-408.

[10] M.L. Yola, V.K. Gupta, T. Eren, A.E. Şen, N. Atar, A novel electro analytical nanosensor based on graphene oxide/silver nanoparticles for simultaneous determination of quercetin and morin, Electrochim. Acta 120 (2014) 204-211.

[11] V.K. Gupta, N. Atar, M.L. Yola, M. Eryılmaz, H. Torul, U. Tamer, I.H. Boyacı, Z. Üstündağ, A novel glucose biosensor platform based on Ag@AuNPs modified graphene oxide nanocomposite and SERS application, J. Colloid Interf. Sci. 406 (2013) 231-237.

[12] V.K. Gupta, M.L. Yola, M.S. Qureshi, A.O. Solak, N. Atar, Z. Üstündağ, A novel impedimetric biosensor based on graphene oxide/gold nanoplatform for detection of DNA arrays, Sensor. Actuat. B-Chem. 188 (2013) 1201-1211.

[13] V.K. Gupta, N. Atar, M.L. Yola, Z. Üstündağ, L. Uzun, A novel magnetic Fe@Au core-shell nanoparticles anchored graphene oxide recyclable nanocatalyst for the reduction of nitrophenol compounds, Water Res. 48 (2014) 210-217.

[14] M.L. Yola, N. Atar, Z. Üstündağ, A.O. Solak, A novel voltammetric sensor based on p-aminothiophenol functionalized graphene oxide/gold nanoparticles for determining quercetin in the presence of ascorbic acid, J. Electroanal. Chem. 698 (2013) 9-16.

[15] M.L. Yola, N. Atar, T. Eren, H. Karimi-Maleh, S. Wang, Sensitive and selective determination of aqueous triclosan based on gold nanoparticles on polyoxometalate/reduced graphene oxide nanohybrid, RSC Adv. 5 (2015) 65953-65962.

[16] N. Atar, M.L. Yola, T. Eren, Sensitive determination of citrinin based on molecular imprinted electrochemical sensor, Appl. Surf. Sci. 362 (2016) 315-322.

[17] M.L. Yola, T. Eren, N. Atar, A sensitive molecular imprinted electrochemical sensor based on gold nanoparticles decorated graphene oxide: Application to selective determination of tyrosine in milk, Sensor. Actuat. B-Chem. 210 (2015) 149-157.

[18] N. Atar, T. Eren, B. Demirdögen, M.L. Yola, M.O. Çağlayan, Silver, gold, and silver@gold nanoparticle-anchored I-cysteine-functionalized reduced graphene oxide as electrocatalyst for methanol oxidation, Lonics 21 (2015) 2285-2293.

[19] N. Atar, T. Eren, M.L. Yola, Ultrahigh capacity anode material for lithium ion battery based on rod gold nanoparticles decorated reduced graphene oxide, Thin Solid Films 590 (2015) 156-162.

[20] Y. Zhang, J. Zhang, H. Wu, S. Guo, J. Zhang, Glass carbon electrode modified with horseradish peroxidase immobilized on partially reduced graphene oxide for detecting phenolic compounds, J. Electroanal. Chem. 681 (2012) 49-55.

[21] J. Zhang, H. Yang, G. Shen, P. Cheng, J. Zhang, S. Guo, Reduction of Graphene Oxide via L-ascorbic 
Acid, Chem. Commun. 46 (2010) 1112-1114.

[22] S. Stankovich, D.A. Dikin, R.D. Piner, K.A. Kohlhaas, A. Kleinhammes, Y. Jia, Y. Wu, S.T. Nguyen, R.S. Ruoff, Synthesis of graphene-based nanosheets via chemical reduction of exfoliated graphite oxide, Carbon 45 (2007) 1558-1565.

[23] X. Zhou, J. Zhang, H. Wu, H. Yang, J. Zhang, S. Guo, Reducing Graphene Oxide via Hydroxylamine: A Simple and Efficient Route to Graphene, J. Phys. Chem. C. 115 (2011) 11957-11961.

[24] S.Y. Toh, K.S. Loh, S.K. Kamarudin, W.R.W. Daud, Graphene production via electrochemical reduction of graphene oxide: Synthesis and characterisation, Chem. Eng. J. 251 (2014) 422-434.

[25] D. Zhang, X. Ouyang, J. Ma, L. Li, Y. Zhang, Electrochemical Behavior and Voltammetric Determination of Curcumin at Electrochemically Reduced Graphene Oxide Modified Glassy Carbon Electrode, Electroanal. 28 (2016) 749-756.

[26] W. Qiao, Y. Li, L. Wang, G. Li, J. Li, B. Ye, Electrochemical behavior of daphnetin and its sensitive determination based on electrochemically reduced graphene oxide modified electrode, J. Electroanal. Chem. 749 (2015) 68-74.

[27] X. Zhu, J. Xu, X. Duan, L. Lu, K. Zhang, Y. Yu, H. Xing, Y. Gao, L. Dong, H. Sun, T. Yang, Controlled synthesis of partially reduced graphene oxide: Enhance electrochemical determination of isoniazid with high sensitivity and stability, J. Electroanal. Chem. 757 (2015) 183-191.

[28] G. Liu, Y. Wang, D. Sun, Investigation of conductivity and catalytic ability at an electrochemically reduced graphene oxide film modified electrode, J. Mater. Sci-Mater. EL. 26 (2015) 943-949.

[29] S.Y. Toh, K.S. Loh, S.K. Kamarudin, W.R.W. Daud, The impact of electrochemical reduction potentials on the electrocatalytic activity of graphene oxide toward the oxygen reduction reaction in an alkaline medium, Electrochim. Acta 199 (2016) 194-203.

[30] B. Li, G. Pan, N.D. Avent, R.B. Lowry, T.E. Madgett, P.L. Waines, Graphene electrode modified with electrochemically reduced graphene oxide for label-free DNA detection, Biosens. Bioelectron. 72 (2015) 313-319.

[31] K. Hamsawahini, P. Sathishkumar, R. Ahamad, A.R.M. Yusoff, A sensitive, selective and rapid determination of lead(II) ions in real-life samples using an electrochemically reduced graphene oxide-graphite reinforced carbon electrode, Talanta 144 (2015) 969-976.

[32] W. Sun, Y. Lu, Y. Wu, Y. Zhang, P. Wang, Y. Chen, G. Li, Electrochemical sensor for transgenic maize MON810 sequence with electrostatic adsorption DNA on electrochemical reduced graphene modified electrode, Sensor. Actuat. B-Chem. 202 (2014) 160-166.

[33] D.A.C. Brownson, L.J. Munro, D.K. Kampouris, C.E. Banks, Electrochemistry of graphene: not such a beneficial electrode material?, RSC Adv. 1 (2011) 978.

[34] M. Velicky, D.F. Bradley, A.J. Cooper, E.W. Hill, I.A. Kinloch, A. Mishchenko, K.S. Novoselov, H.V. Patten, P.S. Toth, A.T. Valota, S.D. Worrall, R.A.W. Dryfe, Electron Transfer Kinetics on Mono- and Multilayer Graphene, ACS nano 8 (2014) 10089-10100.

[35] P.M. Hallam, C.E. Banks, Quantifying the electron transfer sites of graphene, Electrochem. Commun. 13 (2011) 8-11.

[36] J.-H. Zhong, J. Zhang, X. Jin, J.-Y. Liu, Q. Li, M.-H. Li, W. Cai, D.-Y. Wu, D. Zhan, B. Ren, Quantitative Correlation between Defect Density and Heterogeneous Electron Transfer Rate of Single Layer Graphene, J. Am. Chem. Soc. 136 (2014) 16609-16617.

[37] A.G. Güell, N. Ebejer, M.E. Snowden, J.V. Macpherson, P.R. Unwin, Structural Correlations in Heterogeneous Electron Transfer at Monolayer and Multilayer Graphene Electrodes, J. Am. Chem. Soc. 134 (2012) 7258-7261. 
[38] Y. Xu, M. Cao, H. Liu, X. Zong, N. Kong, J. Zhang, J. Liu, Electron transfer study on graphene modified glassy carbon substrate via electrochemical reduction and the application for tris $\left(2,2^{\prime}\right.$ -bipyridyl)ruthenium(II) electrochemiluminescence sensor fabrication, Talanta 139 (2015) 6-12.

[39] L. Chen, Y. Tang, K. Wang, C. Liu, S. Luo, Direct electrodeposition of reduced graphene oxide on glassy carbon electrode and its electrochemical application, Electrochem. Commun. 13 (2011) 133-137.

[40] H.-L. Guo, X.-F. Wang, Q.-Y. Qian, F.-B. Wang, X.-H. Xia, A Green Approach to the Synthesis Graphene Nanosheets, ACS Nano 3 (2009) 2653-2659.

[41] P. Olejnik, A. Świetlikowska, M. Gniadek, B. Pałys, Electrochemically Reduced Graphene Oxide on Electrochemically Roughened Gold as a Support for Horseradish Peroxidase, J. Phys. Chem. C 118 (2014) 29731-29738.

[42] J. Li, C. Chen, J. Wei, J. Li, X. Wang, Enhanced Electrochemical Performance of Reduced Graphene Oxides by H2/Ar Plasma Treatment, J. Phys. Chem. C 118 (2014) 28440-28447.

[43] X. Ji, C.E. Banks, A. Crossley, R.G. Compton, Oxygenated Edge Plane Sites Slow the Electron Transfer of the Ferro-/Ferricyanide Redox Couple at Graphite Electrodes, ChemPhysChem 7 (2006) 1337-1344.

[44] C. Liu, K. Wang, S. Luo, Y. Tang, L. Chen, Direct Electrodeposition of Graphene Enabling the One-Step Synthesis of Graphene-Metal Nanocomposite Films, Small 7 (2011) 1203-1206.

[45] D.K. Kampouris, C.E. Banks, Exploring the physicoelectrochemical properties of graphene, Chem. Commun. 46 (2010) 8986-8988.

[46] R.J. Klingler, J.K. Kochi, Electron-transfer kinetics from cyclic voltammetry. Quantitative description of electrochemical reversibility, J. Phys. Chem. 85 (1981) 1731-1741.

[47] H. Jeong-Yuan, K. Chun-Chiang, C. Li-Chyong, C. Kuei-Hsien, Correlating defect density with carrier mobility in large-scaled graphene films: Raman spectral signatures for the estimation of defect density, Nanotechnology, 21 (2010) 465705. 


\section{Figure captions}

Fig. $1 \mathrm{CVs}$ of GO/GCE in 0.1 M PBS (pH=9.23) at $50 \mathrm{mV} \mathrm{s}^{-1}$. (a) $\mathrm{GO}=0.6 \mu \mathrm{g}$, (b) $\mathrm{GO}=3 \mu \mathrm{g}$, (c) $\mathrm{GO}=6$ $\mu \mathrm{g}$, and (d) $\mathrm{GO}=12 \mu \mathrm{g}$.

Fig. 2 High-resolution deconvoluted C1s XPS spectra of (a) GO and (b-e) ERGOs produced by electrochemical reduction of different masses of GO.

Fig. 3 (a) SEM image of ERGO/GCE. (b) Raman spectra of GO and ERGOs produced by electrochemical reduction of different masses of GO.

Fig. $4 \mathrm{CV}$ results of $1 \mathrm{mM}\left[\mathrm{Fe}(\mathrm{CN})_{6}\right]^{3-/ 4-}$ and $0.1 \mathrm{M} \mathrm{KCl}$ solution on different ERGO/GCEs at a scan rate of $50 \mathrm{mV} \mathrm{s}^{-1}$.

Fig. $5 \mathrm{CV}$ results depicting electrophoretic deposition of graphene in $0.1 \mathrm{M}$ PBS (pH=9.23) with different concentrations of $\mathrm{GO}$ at $50 \mathrm{mVs}^{-1}$ (a) without $\mathrm{GO}$, (b) $\mathrm{GO}=0.1 \mathrm{mg} / \mathrm{mL}$, (c) $\mathrm{GO}=0.5 \mathrm{mg} / \mathrm{mL}$, and (d) $\mathrm{GO}=1 \mathrm{mg} / \mathrm{mL}$.

Fig. 6 High-resolution deconvoluted $\mathrm{C} 1 s$ XPS spectra of ERGOs produced by electrophoretic deposition with different GO concentrations.

Fig. 7 SEM images of ERGO-GCE prepared using (a) $0.1 \mathrm{mg} / \mathrm{mL} \mathrm{GO,} \mathrm{(b)} 0.5 \mathrm{mg} / \mathrm{mL}$ GO, and (c) 1.0 $\mathrm{mg} / \mathrm{mL}$ GO. (d) Raman spectra of GO and ERGOs produced by electrophoretic deposition with different GO concentrations.

Fig. $8 \mathrm{CV}$ results of $1 \mathrm{mM}\left[\mathrm{Fe}(\mathrm{CN})_{6}\right]^{3-/ 4-}$ and $0.1 \mathrm{M} \mathrm{KCl}$ solution on different electrodes at a scan rate of $50 \mathrm{mV} \mathrm{s}^{-1}$. (a) ERGO-GCE and (b) ERGO-modified GCE produced using different methods. 

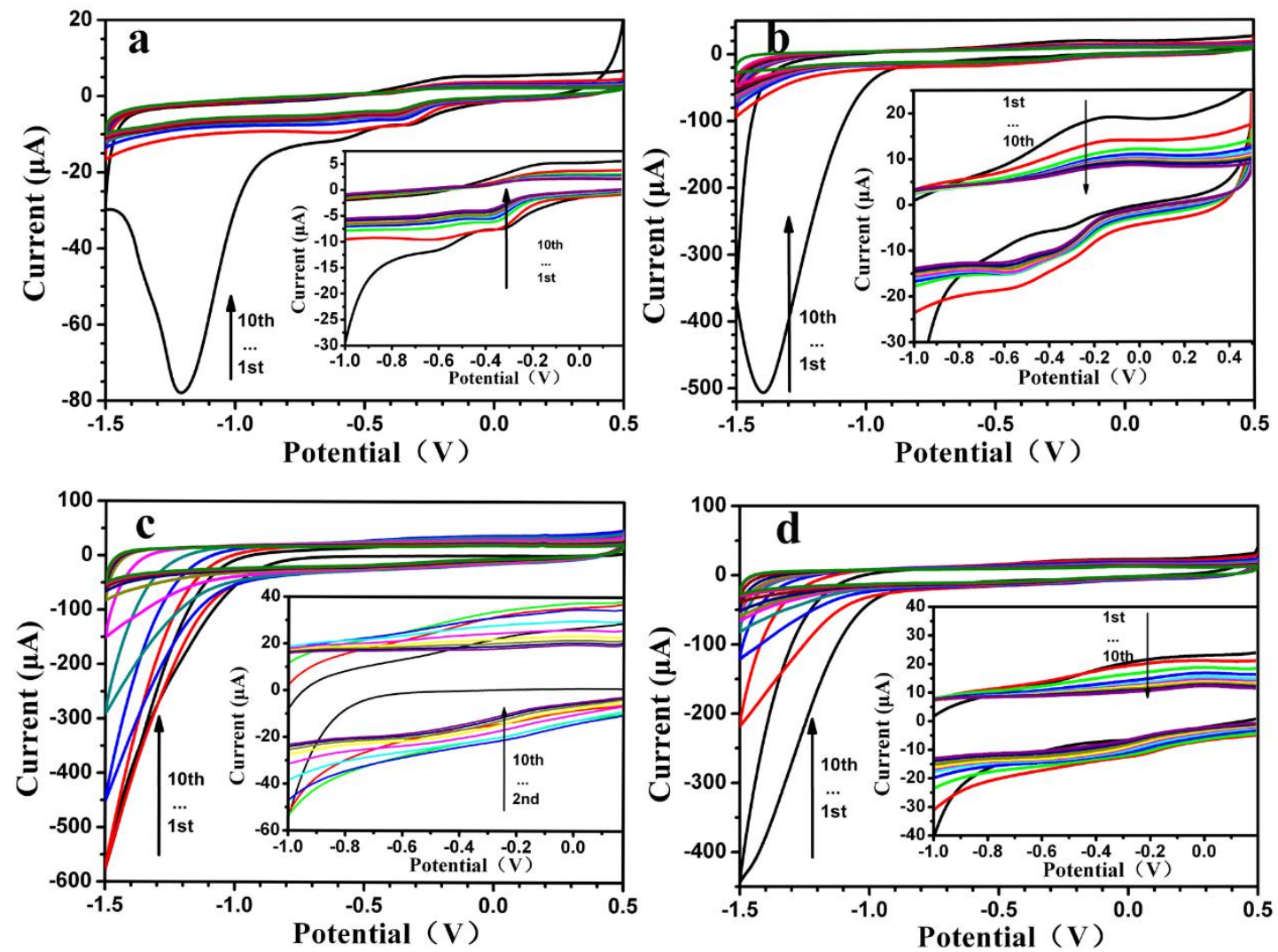

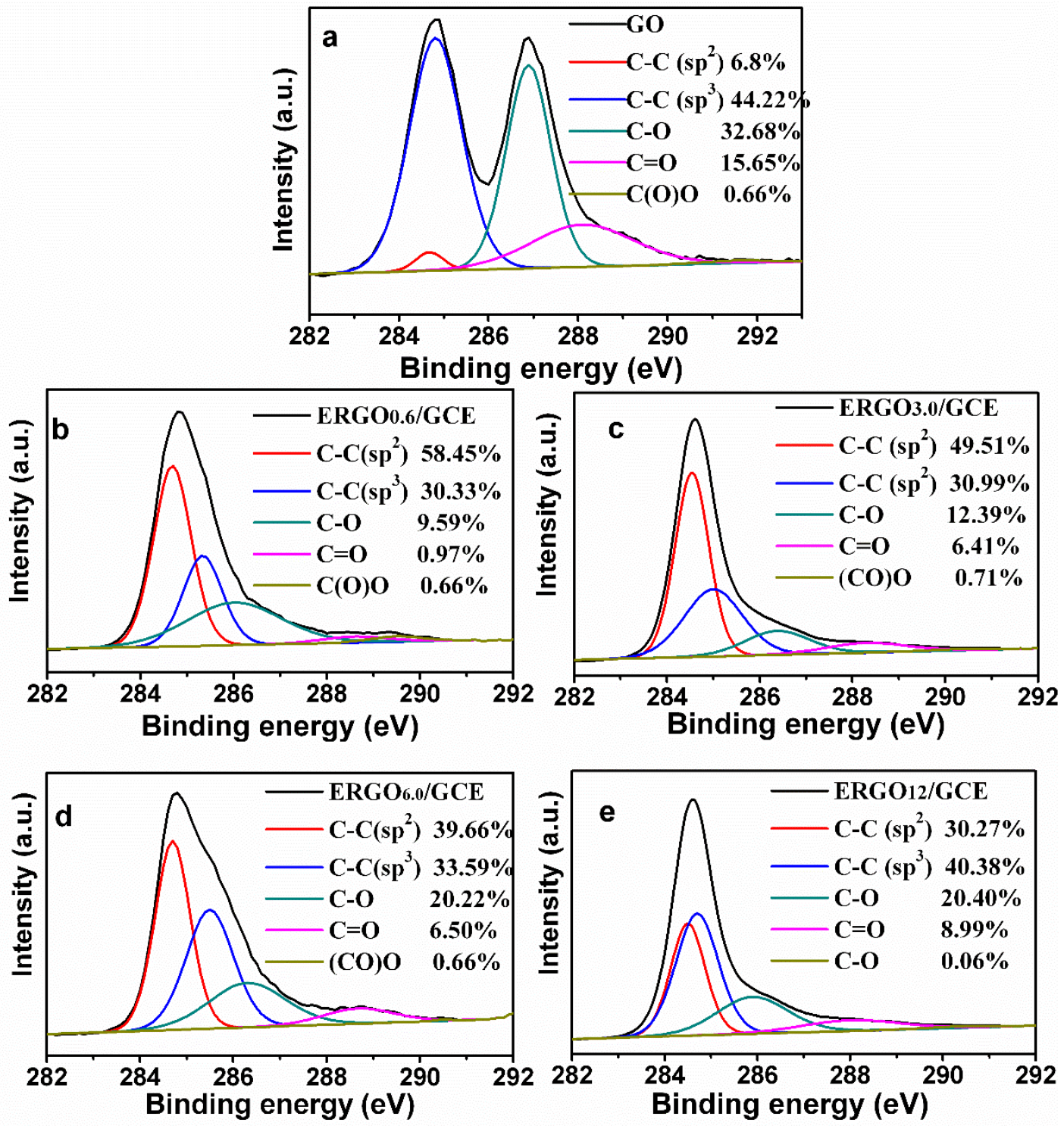

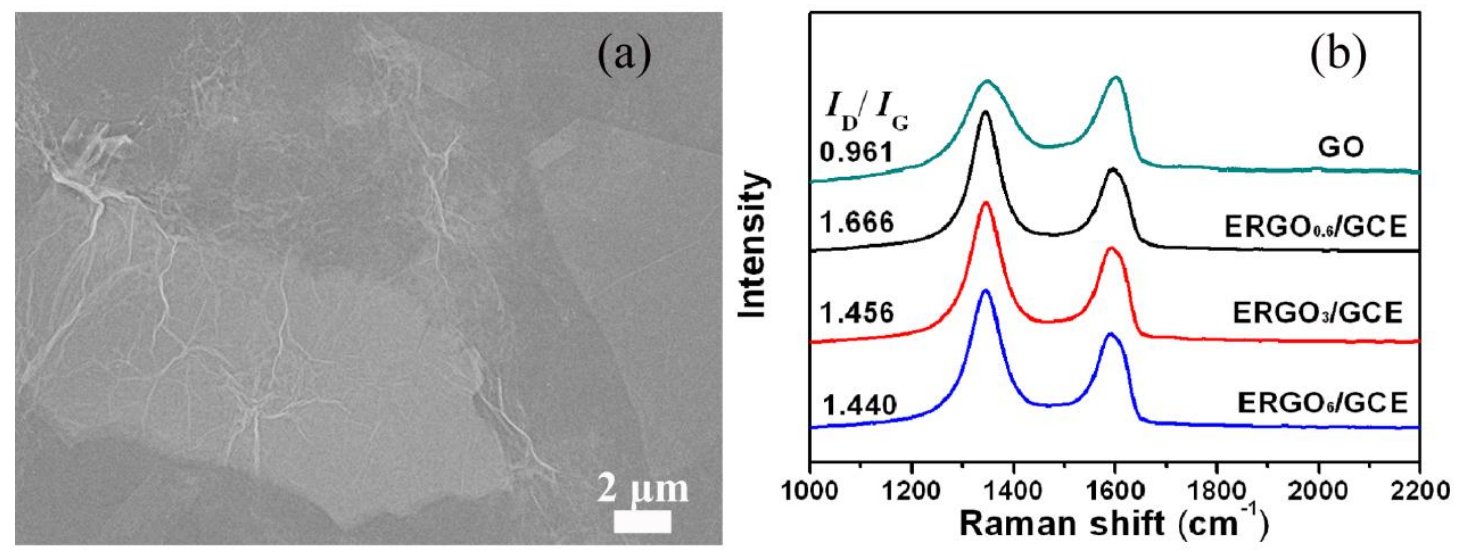


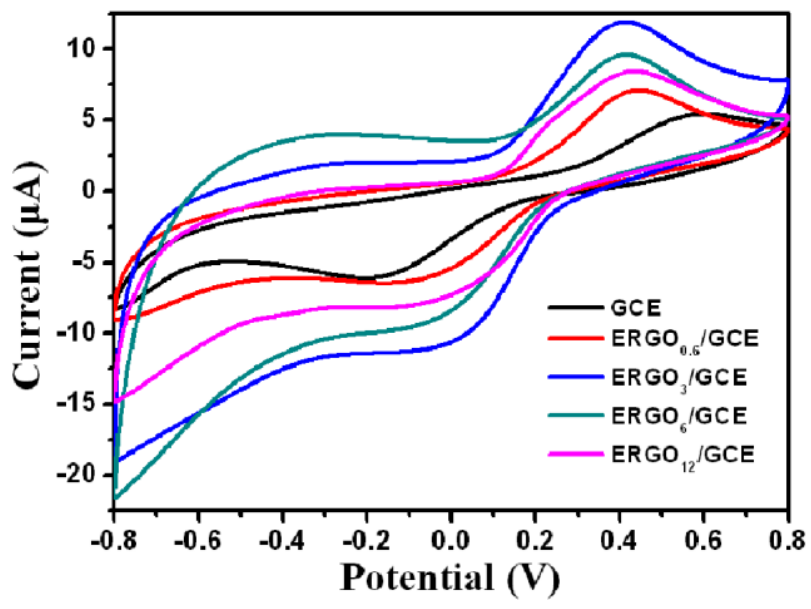



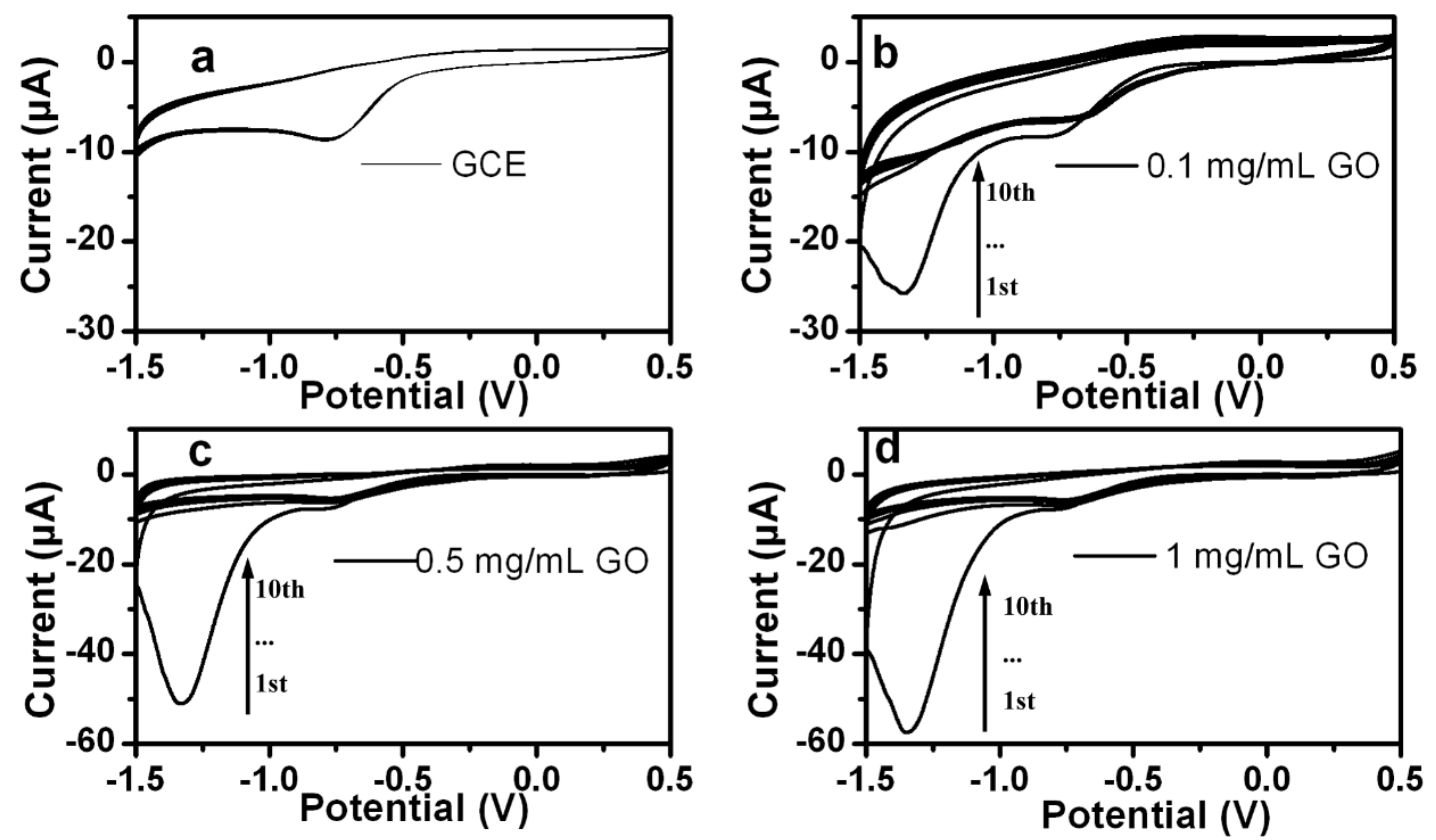

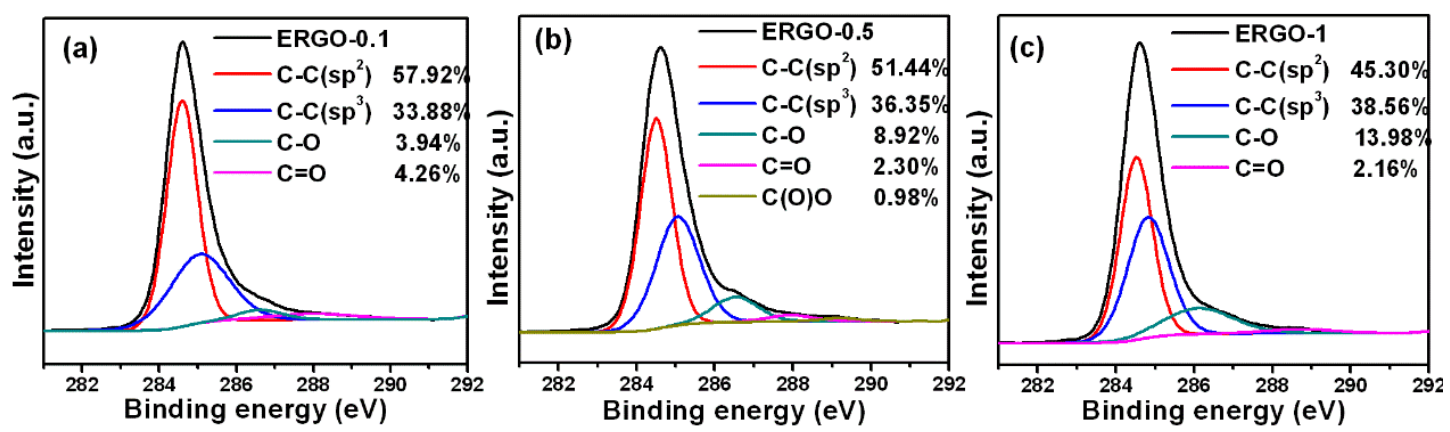

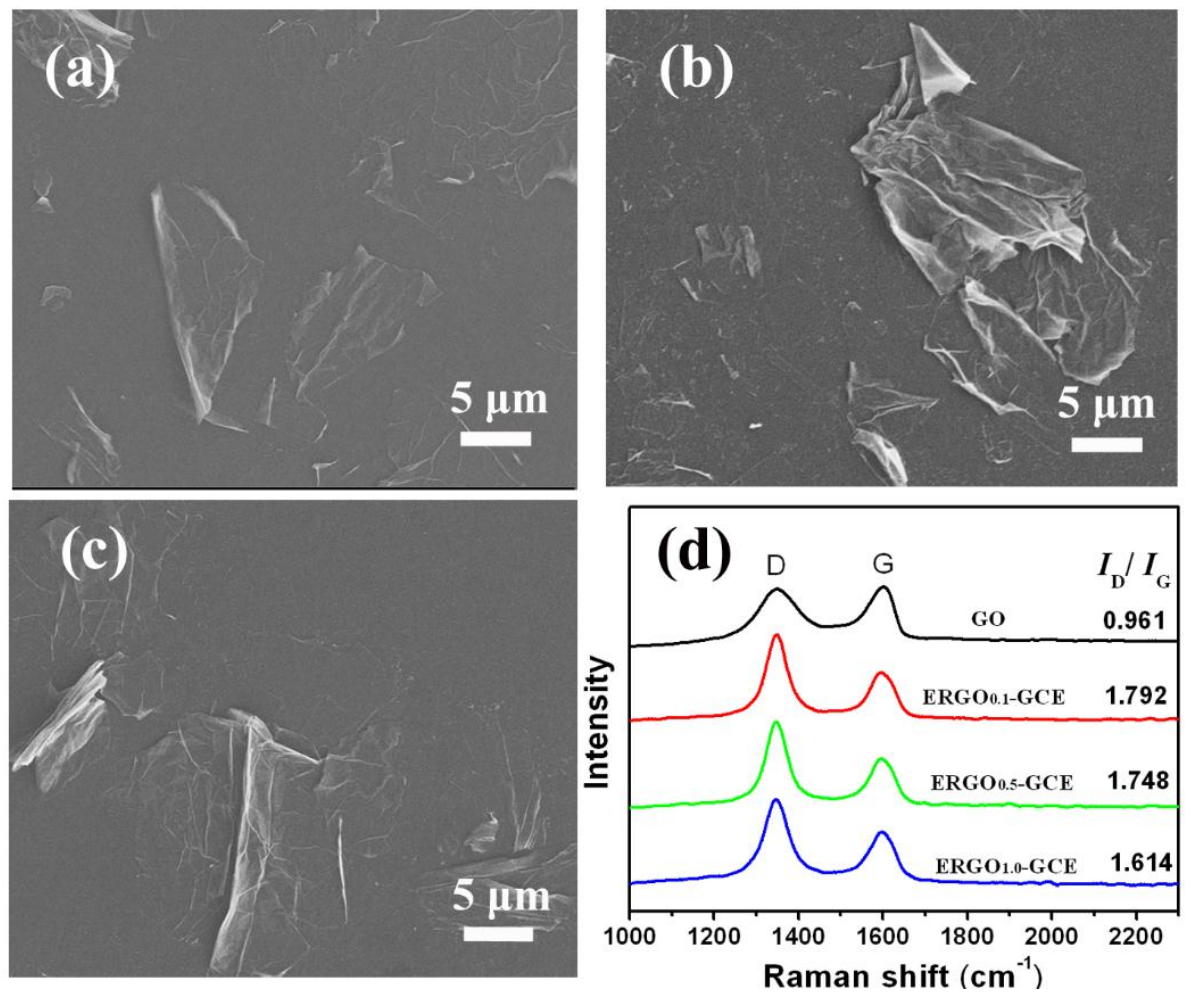

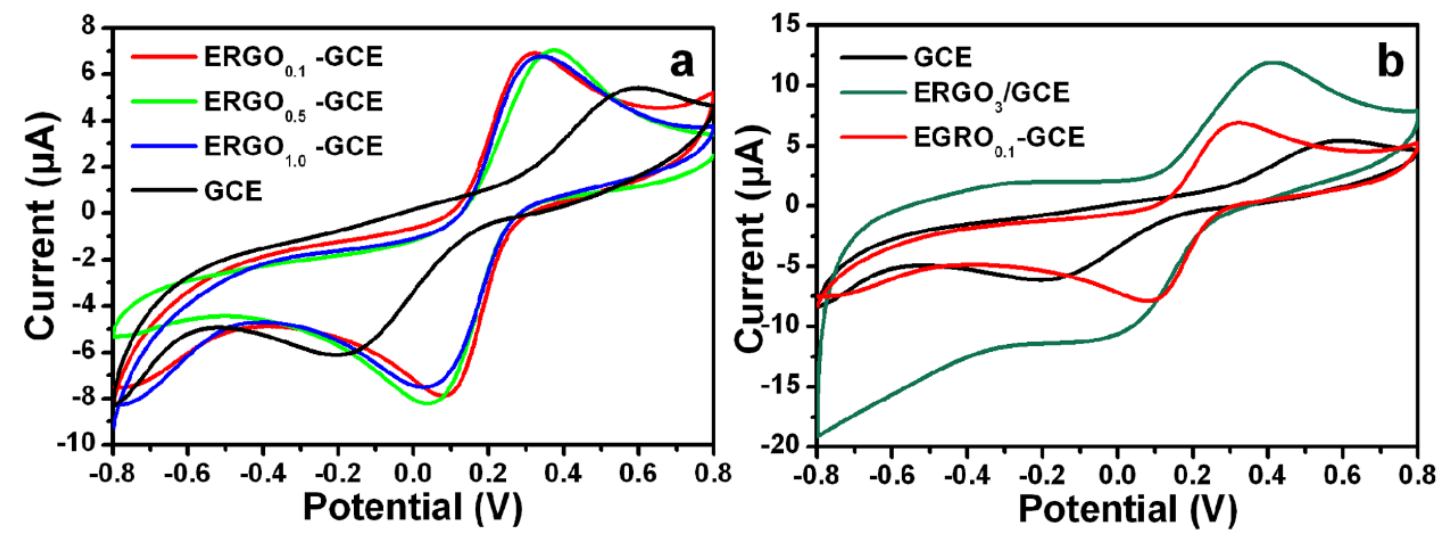